\title{
Guest-Editorial
}

\section{Special issue on the Design of Intelligent Environment}

\author{
Toyohide Watanabe ${ }^{\mathrm{a}, *}$ and Lakhmi C. Jain ${ }^{\mathrm{b}}$ \\ ${ }^{a}$ Department of Systems and Social Informatics, Graduate School of Information Science, Nagoya University. \\ Furo-cho, Chikusa-ku, Nagoya 464-8603, Japan \\ ${ }^{\mathrm{b}}$ School of Electrical and Information Engineering, University of South Australia, Mawson Lakes Campus, South \\ Australia 5095, Australia
}

When we analyse behaviour from the viewpoint of information technologies, computer-supported systems may be mainly divided into two types of functional mechanisms. There are processing means and communication means. These two systems sort our activities from the view of Function and Mechanism. This special issue focuses on the correspondence between persons and computers or information systems and from the viewpoint of service support. It also investigates the use of information technologies in a wide range of application fields. This is done in terms of the current state-of-art. This volume contains eight research papers dealing with the theory and applications in a computersupported behaviour environment. These eight papers may be divided into four groups. These are the Computer Support for personal activities in the first two papers and Computer Support for an E-learning Environment in the next two papers. Computer Support for Information Networks is dealt with in a successive paper. Finally Computer Support for Social Simulation is covered in the remaining three papers.

The first paper by Watanabe and others focuses on extracting the information exchanged in collaborative learning process. It provides knowledge for the solution of the subjects discussed. It organizes the knowledge structure into a usable form. This usable

\footnotetext{
${ }^{*}$ Corresponding author. E-mail: watanabe@is.nagoya-u.ac.jp.
}

knowledge structure is called the Solution Knowledge Graph. The computer function automatically organizes the knowledge into a suitable structure.

The second paper by Yatsugi and others proposes to manage and utilize the photographs into form suitable for the user's interests. Slide-show of personal photographs is made automatically for user. These photographs have tags. When composing an insight slideshow, the weight of the semantic relation between two photographs is calculated, using these tags and their coincident use in the Web. The computer function organizes a suitable reference structure.

The third paper by Kunimune and others proposes an Web-based learning system which enables these learning systems to write annotations directly onto the learning materials. This can then be shared between lectures and students. This system makes it possible for the students and lectures to discuss the shared memos with any attached comments on the memos. The computer function supports the manipulation of any annotations in a learning environment.

The fourth paper by Nishino and others analyses the learning preferences of the students in e-learning courses. It discusses the adaptabilities of e-learning courses. Using these analyses, an effective e-learning system is produced, which offers learning courses and learning objectives, suitable for individual students. The computer function manages the e-learning courses.

The fifth paper by Mineno and others describes a remote information sensing system, applied to a dense 
sensor network. This is applied to agricultural management used in melon hothouses. Sensor networks are critical tools when measuring and understanding complex interactive dynamics. This paper reports some experimental results of the performance and is also applied to the design of such a system. The computer function controls the collective sensing data analytically and computes these results.

The sixth paper by Hasegawa and others proposes an autonomous access point selection algorithm for use with user-centric radio resource usage optimization in distributed wireless networks. This optimisation algorithm is based on the mutually connected neural network. This minimizes a given objective function by the use of a distributed update for each neuron. The computer simulation shows that the proposed algorithm obtains the best available throughput for each of the users in a large-scale wireless network.

The seventh paper by Takamiya and others proposes a method of estimating travel behaviours. It uses Zone Characteristics which are obtained from the existing city-structured data. This method does not require survey data concerning any new travel behaviour. On this method, Bayesian Network analysis is applicable, using individual person trip data and city-structured data. The computer simulation is applied to analytically evaluate the performance results.

The eighth paper by Mukai amd others focuses on a new transport system "On-Demand Bus System”. This is introduced to local cities using a trial basis. Electrical vehicles are considered as the On-Demand Buses. The Path Optimization Algorithm is based on an evolutionary approach. It is investigated as The Research Objective. It minimizes the travelling distance of vehicles so as to reduce the recharge time. The computer simulation is used to make the performance explicit in which is contrast to other algorithms.

All papers are visionary and should be appropriated by the scientific community. We thank reviewers for their constructive feedback.

Toyohide Watanabe, Japan

Lakhmi C. Jain, Australia 